\title{
A prática do auto-exame das mamas em mulheres de uma comunidade universitária
}

\author{
Marisa Campio Müller \\ Antônio Frasson \\ Chana Kieling \\ Fernanda Silva Hoffmann \\ Patrícia Fleck \\ Hericka Zogbi \\ Adriana Zanona da Matta \\ Joyce Werres
}

\begin{abstract}
Resumo
Dados estatísticos atuais apontam para elevados índices de prevalência e mortalidade em mulheres acometidas pelo câncer de mama (INCA, 2004), o que poderia ser minimizado pela prática do auto-exame, visto que esta se caracteriza como elemento facilitador de diagnóstico precoce. Esta pesquisa teve como objetivo conhecer a prática do auto-exame das mamas em mulheres representantes da população da PUCRS. Para tanto foi utilizada uma abordagem mista (qualitativa e quantitativa), aplicando-se como instrumento um questionário semi-estruturado, em uma amostra de 215 mulheres. Os resultados mostraram que apenas 25\% das mulheres pesquisadas referem adotar a prática do auto-exame das mamas periodicamente como cuidado preventivo. $O$ estudo também evidenciou que todas essas mulheres reconhecem a importância desta prática, porém, muitas vezes não sabem ou não lembram de realizá-la, e outras vezes a praticam de maneira inadequada (com relação à freqüência).

Palavras-Chave: Auto-exame; Câncer de mama; Diagnóstico precoce.
\end{abstract}

\section{Breast self-exam practice in women from an universitary community}

\begin{abstract}
Current statistical data point to high rates of prevalence and death among women undergoing breast cancer (INCA, 2004). This issue could be minimized through the self exam practice, since this procedure is considered a facilitating element in early diagnosis. This research aimed at knowing the practice of breast self-exam in women, representing the population of PUCRS, wich is a university. A mixed methodology was used (quantitative and qualitative) and as a tool, semi-structural questionnaire was applied in a sample of 215 women. The results show that only 25 percent of researched women refer to the habit of practicing breast self-exam periodically as a preventive care. The study also enhanced that all women recognize the importance of this practice however, many times they don't know how to do it or don't remember to do it and many other times they do it in an inappropriate way (related to frequency). Keywords: Self-exam; Breast of cancer; Early diagnosis.
\end{abstract}

\section{Introdução}

O câncer de mama é uma doença que vem se tornando cada vez mais freqüente entre as mulheres. De acordo com o Instituto Nacional de Câncer (INCA, 2004), esse tipo de câncer é o mais comum entre as mulheres no contexto mundial. No Rio Grande do Sul, além da alta prevalência de pessoas acometidas por este tipo de câncer, observa-se uma alta taxa de mortalidade, a qual se deve, em grande parte, ao avançado estágio da doença encontrado no momento do diagnóstico.

Segundo Novais (2004), presidente da Sociedade Brasileira de Mastologia, 70\% dos casos podem ser detectados pela apalpação. Além de detectar doenças malignas e benignas na mama (mas que necessitam de tratamento), o auto-exame também contribui para que as mulheres que não têm acesso fácil aos serviços de saúde sejam motivadas a procurá-los em casos alterados.

Alguns pesquisadores (Marinho, Costa-Gurgel, Cecatti \& Osis, 2003; Tubiana \& Koscielny, 1999), apontam que, pelo diagnóstico precoce, é possível reduzir a mortalidade por câncer de mama, visto que este pode identificar o tumor com o menor tamanho possível e em seu estágio inicial.

$\mathrm{O}$ auto-exame caracteriza-se como um processo simples e indolor que auxilia na detecção do câncer em seu estágio inicial, podendo esse aparecer na forma de pequenos nódulos nas mamas. A realização correta do

${ }^{1}$ Endereço para correspondência:

Av. Ipiranga, 6.681, Pd. 11, 9o andar, sala 938 - 90619-900 - Porto Alegre-RS

E-mail: gpps938@hotmail.com 
auto-exame dá-se uma vez ao mês, durante a segunda semana após a menstruação. É importante salientar que à realização fora deste período poderá detectar falsas impressões. Uma descoberta deste tipo deve levar à procura de um especialista, o mais breve possível, a fim de evitar maiores danos, facilitar o tratamento e, possivelmente, a cura (Frasson, 2000).

Observações assistemáticas de profissionais da saúde reconhecem que muitas mulheres não praticam esse procedimento. Estas observações motivaram-nos a realização desta pesquisa, a qual teve como objetivo conhecer a prática do auto-exame das mamas em mulheres da população da Pontifícia Universidade Católica do Rio Grande do Sul (PUCRS). Além disso, este estudo teve também o intuito de divulgar a importância da prevenção do câncer de mama, que vem assumindo grande impacto social.

Embora a mamografia seja amplamente considerada como um método eficaz na deteç̧ão precoce do câncer de mama, apenas uma pequena porcentagem deste tipo de câncer é detectado neste procedimento (American Câncer Society, 2004; Epstein, Bertell \& Seaman, 2001). Desta forma, o auto-exame se torna um método de detecção precoce simples e de baixo custo, devendo estar associada a exames clínicos (Norman \& Brain, 2005).

Através desses objetivos buscamos refletir sobre o uso ou a negligência desse método de diagnóstico precoce como também sobre os estigmas envolvidos nesta doença. Acreditamos ser dever da sociedade e dos profissionais da saúde incentivarem a informação, o combate e a prevenção da doença, visto que a bibliografia pesquisada mostra que muitas mulheres não realizam o auto-exame, mesmo sendo este um procedimento bastante simples (Pacheco, Botega \& Silveira, 1996; Marinho, Costa-Gurgel, Cecatti \& Osis, 2003).

A realização da prevenção por meio do autoexame também implica o conhecimento das mulheres sobre seu corpo. A detecção de alguma anormalidade, no momento do auto-exame, é facilitada quando as mulheres já apresentam uma certa intimidade com o mesmo. Nos casos em que este procedimento não ocorre, o câncer acaba sendo descoberto num estágio mais avançado, necessitando muitas vezes de uma intervenção mais evasiva, como a retirada de um quadrante da mama ou até mesmo de toda a mama. Uma intervenção dessa magnitude pode trazer um desequilibrio emocional muito grande na vida da mulher, visto que se refere ao seio, órgão que traz embutidos, além da questão da saúde, aspectos ligados à feminilidade, beleza e sensualidade da mulher.

\section{Método}

A presente pesquisa tratou-se de um estudo descritivo, transversal, de abordagem mista. Este tipo de abordagem, conforme Víctora, Knauth e Hassen (2000), permite uma complementaridade entre os métodos qualitativo e quantitativo, possibilitando com isso uma integração de dados dentro de um mesmo projeto.

Este estudo foi composto por uma amostra de 215 mulheres, na faixa etária dos 17 a 63 anos, professoras, funcionárias e universitárias da PUCRS.

Como instrumento utilizou-se um questionário semi-estruturado, auto-aplicável, com perguntas fechadas e abertas, elaborado pelos pesquisadores com base na revisão bibliográfica realizada. A aplicação deste instrumento deu-se somente após a concordância das participantes com o Termo de Consentimento Livre e Esclarecido.

Os dados obtidos através das questões abertas foram analisados pelo método de análise de conteúdo de Bardin (1977). Já as respostas fechadas do questionário foram analisadas quantitativamente, por análise de freqüência simples e de correlação estatística.

Todo o processo deu-se de forma sigilosa, preservando a identidade das participantes.

\section{Resultados}

A amostra caracterizou-se por mulheres, das quais $53 \%$ estudantes, $36 \%$ professoras e $11 \%$ funcionárias da PUCRS.

A maioria das entrevistadas caracteriza-se por serem estudantes, entre 17 e 29 anos (56\%), solteiras $(60 \%)$ e cursando a área de Ciências Humanas (62\%).

Quanto à questão que investigou sobre o que é o câncer de mama, $98 \%$ da amostra referiu saber o que é esta doença, e destas, $53 \%$ a definiram como um nódulo e $79 \%$ identificaram-no como maligno.

A grande maioria das participantes $(87 \%)$ mencionou saber como se trata o câncer de mama, e 34\% destas citaram como tratamento a quimioterapia, $24 \%$ a cirurgia, $23 \%$ a radioterapia e $6 \%$ a mastectomia. Quando perguntada sobre quais as conseqüências do câncer de mama, 94\% referiram sabê-las. Estas mulheres obtiveram as informações acerca do câncer de mama através dos meios de comunicação (43\%), dos quais os de maior abrangência foram jornais e revistas (27\%), e do diálogo entre amigos, família e/ou conhecidos (39\%).

Quanto à abrangência do câncer de mama nesta população, $68 \%$ das mulheres mencionaram conhecer alguma pessoa que já teve ou tem a doença, em $49 \%$ das vezes havendo um grau de parentesco entre elas (mãe, irmã e tia).

Quanto aos cuidados adotados, $86 \%$ responderam fazer algum tipo de prevenção. Dentre os cuidados preventivos mais utilizados estão as visitas regulares ao médico (29\%), a mamografia (14\%) e o auto-exame das mamas periodicamente $(25 \%)$. Verificamos, então, que é relativamente baixo o número de mulheres que realiza o auto-exame das 
mamas periodicamente, apesar de a maioria delas (91\%) referirem já tê-lo feito pelo menos uma vez na vida.

Todas as participantes responderam achar necessária a realização do auto-exame e, quando questionadas sobre o porquê desta necessidade, $44 \%$ responderam que seria para identificar a doença e $45 \%$ responderam que seria para prevenir ou para tratar o câncer de mama.

As informações sobre como realizar o autoexame foram obtidas através dos meios de comunicação (48\%), dos médicos e/ou enfermeiras (28\%), dos amigos e/ ou familiares (15\%), dentre outros.

Quanto à freqüência da realização do auto-exame, $49 \%$ dizem fazê-lo uma vez ao mês, $26 \%$ o realizam uma ou duas vezes ao ano e $12 \%$ fazem o auto-exame uma vez por semana ou todos os dias, apenas $13 \%$ referindo não realizar o auto-exame. Dentre as mulheres que não o realizam e as que o realizam com freqüência incorreta, elas justificam tal atitude pelo esquecimento em 32\% dos casos, $21 \%$ pelo medo de encontrar algo ou de sentir dor, $13 \%$ por não saber como fazê-lo, $8 \%$ por não ter o hábito de se tocar, $6 \%$ por achar que o auto-exame não é importante e $3 \%$ por achar que estão livres dessa doença.

A maioria das mulheres participantes desta pesquisa relatou sentir-se confortável em relação ao tocar o corpo (97\%). Destas, $48 \%$ ainda acrescentam a importância do tocar-se/conhecer-se, e apenas 3\% mencionam ter preconceitos em relação ao toque.

Quando questionadas sobre o que fariam caso fossem diagnosticadas com câncer de mama, as mulheres responderam que procurariam um tratamento médico adequado (39\%), recursos externos como ajuda psicológica, técnicas alternativas e ajuda de amigos (20\%), 16\% sentiriam um grande sofrimento emocional e $10 \%$ referiram que buscariam apoio em seus recursos internos.

Essas mulheres acreditam que os aspectos emocionais podem influenciar no desencadeamento do câncer de mama $(85 \%)$, sendo esta resposta estatisticamente mais significativa para as professoras $(p<0,01)$ e mulheres na faixa etária acima de 50 anos $(p<0,01)$. Entre esses aspectos, os mais apontados foram o estresse $(26 \%)$ seguido da idéia de somatização (18\%), sentimentos negativos como raiva, ódio e angústia (15\%) e a depressão (14\%).

\section{Discussão dos resultados}

Tomando por base os resultados obtidos e a bibliografia revisada percebemos que as mulheres pesquisadas sabem o que é câncer de mama e obtêm essas informações, em sua maioria, através dos meios de comunicação. Referem saber quais as conseqüências do câncer de mama e como tratá-lo. Porém, muitas vezes, mostram não saber ou não lembrar de realizar o autoexame e, quando o realizam, praticam-no de maneira Psico-USF, v. 10, n. 2, p. 185-190, jul./ dez. 2005 incorreta (com relação à freqüência). Esse esquecimento, ou seja, esse "não lembrar" mencionado pelas participantes nos remete ao impacto psicológico causado pelo câncer na vida de uma pessoa, muitas vezes levando-a a uma redefinição da autopercepção sobre a sexualidade e a imagem corporal.

Percebemos que as mulheres entrevistadas reconhecem a importância e a necessidade do autoexame, entretanto, no que se refere à prática do mesmo, mostram uma ação incorreta, mesmo que praticamente a metade desta população tenha parentes que têm ou já tiveram câncer, o que significa que podem ocorrer riscos de desenvolvê-lo, devido ao componente genético. Com isso, percebemos que a proximidade com pessoas que já tiveram câncer de mama não é um requisito para a realização de cuidados preventivos, como o auto-exame.

Outro aspecto relevante a ser comentado quanto à questão do esquecimento é que este é usado como justificativa para a não-realização do auto-exame, evidenciando, talvez mediante uma linguagem corporal, um descuido com o próprio corpo. Esquecer seria uma forma de não entrar em contato com o próprio corpo e, conseqüentemente, com as chances de detectar algo estranho (doença) no mesmo. Contudo, esse esquecimento pode levar a um afastamento dos riscos reais, ao mesmo tempo em que pode servir como uma defesa diante da possibilidade da morte.

Outra forma de pensar essa defesa estaria ligada às questões sexuais, visto que algumas culturas e religiões ainda desaprovam a exploração do próprio corpo. Sendo assim, o auto-exame torna-se uma prática inaceitável por desencadear ansiedades masturbatórias (Pacheco e cols., 1996).

Um estudo realizado por Marinho, CostaGurgel, Cecatti e Osis (2003), em Campinas/SP, teve o objetivo de avaliar o conhecimento, a atitude e a prática do auto-exame das mamas entre usuárias de centros de saúde. As mulheres pesquisadas, em sua maioria, estavam em idade avançada e apresentavam baixa escolaridade. Após um estudo observacional descritivo com 663 mulheres, os pesquisadores chegaram aos seguintes resultados: a amostra apresentou uma atitude adequada e favorável à realização do auto-exame, isto é, as mulheres reconheceram a importância do auto-exame como fator de diagnóstico precoce, no entanto, quanto à prática elas mostraram-se inadequadas. Este resultado mostrouse bastante similar à pesquisa por nós realizada, embora a população estudada tenha sido predominantemente jovem e de alta escolaridade.

Quanto às informações disponíveis relacionadas à prevenção do câncer de mama, pudemos constatar que elas existem, porém, parecem não cumprir sua função preventiva, visto que há problemas na assimilação e 
posterior execução da prática do auto-exame na população pesquisada. Dessa forma, pensamos que as campanhas que se propõem a incentivar a realização do auto-exame podem não estar voltadas para as reais necessidades das mulheres. Estes dados fornecem-nos subsídios para traçar novas campanhas informativas e preventivas, de acordo com o conhecimento ou a falta deste nas mulheres desta população.

Urbanetz, Oliveira e Hatschbach (1992) acrescentam que o ensino da técnica para a realização do auto-exame deve ser de responsabilidade dos profissionais da saúde, durante consultas ginecológicas ou em reuniões educativas.

Aspectos contraditórios com relação à afirmação da prática e da importância do auto-exame podem nos remeter a algumas reflexões sobre o tema. Em algumas questões do questionário as mulheres responderam achar necessária a prática do auto-exame referindo realizá-lo, porém, em outras questões evidenciaram o oposto. Talvez esse achado possa estar relacionado à falta do hábito de posicionamento e reflexão diante do assunto e/ou em razão do medo da possibilidade de encontrar um tumor, o que foi relatado por elas.

Farah (1995) propõe que a simples idéia de que algo em nosso corpo mudará fora de nossa vontade, como a necessidade de retirar uma parte da mama, é muito assustadora. Além disso, essa mudança no corpo implica uma reformulação na mente de sua imagem corporal. Esse temor pode fazer as mulheres agirem de maneira onipotente, preferindo pensar que nada acontecerá a elas. Pensamos que estas idéias podem estar relacionadas com a população, que é relativamente jovem e que ainda possui esse pensamento "mágico", apesar de possuírem uma alta escolaridade. Ou ainda pelo fato de que o câncer de mama, pela prática médica, é considerado raro em mulheres abaixo de 35 anos, o que pode contribuir para essas mulheres acharem que estão livres da doença.

As participantes acreditam que os aspectos emocionais podem influenciar no desencadeamento do câncer de mama, sendo esta resposta estatisticamente mais significativa para as professoras e mulheres na faixa etária acima de 50 anos.

Acreditamos que esse dado possa estar relacionado à maior espiritualidade, experiência de vida e maturidade dessas mulheres nessa faixa etária. Aqui nos cabe citar Jung (1964), quando este refere que na fase da meia idade as pessoas passam a procurar idéias e convicções que dêem sentido a sua vida. Assim, a crença em relação aos aspectos emocionais cumpriria uma função explicativa de sentido da doença, a qual parece não ser satisfeita somente pelos aspectos orgânicos. Isso sugere também o reconhecimento da questão integrativa entre mente e corpo, a qual vem sendo pesquisada e desenvolvida por diversos autores, podendo ser considerada uma idéia atual e desafiadora, principalmente no meio médico.

A prevalência do estresse entre os fatores emocionais citados pelas mulheres desta pesquisa como desencadeadores do câncer de mama pode nos fazer pensar em questões como qualidade de vida e nível atual de exigência sobre a mulher. Há pouco tempo as mulheres passaram a assumir papéis mais representativos na sociedade, como profissionais, levando-as a uma maior liberdade financeira, como também a novas responsabilidades. Pensamos que este momento de transformações de papéis familiares e de novas descobertas pelas mulheres possa estar associado à presença do estresse como um fator psicológico desencadeante de doenças.

\section{Considerações finais}

Muito se tem pesquisado a respeito da relação do ser humano com seu corpo e, mais especificamente, sobre a relação deste com um corpo estranho, no caso do câncer, um tumor. Nos últimos anos o câncer tem sido alvo de diversos estudos e campanhas de prevenção, que nos apontam para novas descobertas farmacológicas. Assim, podemos dizer que consideráveis achados já foram feitos. Contudo, ainda há muito a se desenvolver sobre o tema. Esse campo interminável e inesgotável de possibilidades é que nos fornece subsídios para realizar algumas considerações pertinentes ante o término deste estudo.

Percebemos que o câncer, e aqui falamos mais especificamente do câncer de mama, parece ainda ser uma doença carregada de estigmas e preconceitos, mesmo diante do aumento do índice de sobrevida de pacientes com esse diagnóstico. Desta forma, a idéia de estar com câncer, ou melhor, encontrar um tumor na mama, parece estar ainda muito associada à morte, dor, e, sentimentos negativos.

Este estudo demonstrou que as mulheres pesquisadas referem saber o que é o câncer de mama, revelando conhecimento sobre o tratamento do mesmo. Todavia, no momento de praticá-lo, o fazem de maneira inadequada, o que acaba tornando a prática ineficaz quanto a seu objetivo, que é o diagnóstico precoce.

Diante deste dado, o qual nos parece de muita significância por evidenciar certa discrepância entre teoria e prática, surgem novas possibilidades para pensarmos sobre a relação da mulher com o câncer de mama, ou melhor, com a probabilidade de vir a tê-lo.

As campanhas preventivas podem estar deixando a desejar quanto a sua função de detecção precoce da doença, o que nos leva a pensar em uma necessidade de sua reformulação. Caso essas campanhas fossem reavaliadas, informando com mais clareza os procedimentos para realização eficaz do auto-exame, de acordo 
com as necessidades das mulheres, poderíamos ter um número maior delas realizando adequadamente essa prática. Porém, não podemos ignorar a possibilidade de que, mesmo realizando essas reformulações, o objetivo pode não ser atingido, em virtude da existência de mecanismos psíquicos de negação e do estigma associado ao câncer de mama.

Diversos teóricos, ao longo do tempo, nos trazem que é da natureza humana evitar a dor e negar a morte, pois o homem possui um instinto inato: o instinto de vida, de sobrevivência. A doença viria romper com essa negação, fazendo o homem se deparar com a mortalidade, reconhecendo que não é um ser onipotente, ou seja, que é um ser humano capaz de adoecer, sofrer e morrer. Assim, o temor de encontrar algo estranho na mama pode ser uma das questões que se encontram associadas à não-prática ou à prática inadequada do auto-exame.

Outro aspecto que nos parece relevante sobre a prática do auto-exame diz respeito à relação da mulher com sua sexualidade. Sabemos que a mama é um órgão repleto de simbolismo, representando a feminilidade e a maternidade. Sabemos também que nos tempos atuais a sexualidade é um tema bastante discutido, difundido e valorizado. Todavia, parece que mesmo nessa nova era ainda sofremos influências de um período anterior, mais rígido, sustentado pelo silêncio, pela vergonha e desaprovação da sexualidade. Talvez a falta de prática do auto-exame e/ou sua prática equivocada possa ser um sinalizador dessa confusão atual de valores em que a banalização do sexo pode levar à despreocupação com o corpo quanto à saúde, havendo uma preocupação demasiada com a estética.

Compartilhamos a idéia de que as questões que abrangem a prática do auto-exame das mamas são de ordem complexa, mas possíveis de compreensão, mediante estudos e pesquisas na área. Pensamos que, enquanto nós, profissionais da saúde, estivermos abertos a tentar compreender o ser humano de forma mais integrada, estaremos contribuindo para o conhecimento científico, bem como para novas reflexões e possíveis alternativas sobre o tema.

\section{Agradecimentos}

Agradecemos ao $\mathrm{CNPq}$ pelo financiamento desta pesquisa e incentivo à ciência.

\section{Referências}

American Cancer Society (2004). Can breast cancer be found early? [On-line]. Disponível em: <http://www. cancer.org/docroot/CRI/content/CRI_2_4_3X_Can _breast_cancer_de_found_early_5.asp $>$.
Bardin, L. (1977). Análise de conteúdo. Lisboa: Edições 70.

Epstein, S. A., Bertell, R. \& Seaman, B. (2001). Dangers and unreability of mammography: Breast examination is a safe, effective, and practical alternative. International Journal of Health Services, 31, 605-615.

Farah, R. M. (1995). Integração psicofísica: o trabalho corporal e a psicologia de C. G. Jung. São Paulo: Robe.

Frasson A., Saggin, L. \& Zermes, F. (2000). Prevenção do câncer de mama. Em Mastologia Prática (pp. 15-28). Caxias do Sul: UCS.

INCA (Instituto Nacional de Câncer) (2004). Controle do câncer de mama: documento de consenso. [On-line]. Disponível em: <http://www.inca.gov.br>.

Jung, C. G. (1964). O homem e seus símbolos. Rio de Janeiro: Nova Fronteira.

Karger, L. (2002). Defend yourself against cancer. Female Patient (on-line) OB/GYN ed.; Chatham, Iss.S33, (pp. 2-8). Disponível em: <http://proquest. umi.com/pqdweb?did $=110124233 \&$ sid $=4 \& \mathrm{Fmt}=3 \&$ clientId $=22430 \& R Q T=309 \& V N a m e=P Q D>$.

Marinho, M. S., Costa-Gurgel, L. A. B., Cecatti, J. G. \& Osis, M. J. D. (2003). Conhecimento, atitude e prática do auto-exame das mamas em centros de saúde. Revista de Saúde Pública, 37(5), 576-582.

Norman, P. \& Brain, K. (2005). An application of an extended health belief model to the prediction of breast self examination among women with a family history of breast cancer. British Journal of Health Psychology, 10(1), 1-16.

Novais, E. (2004). Toque não evita morte por câncer de mama (on-line). Disponível em: <http://www.netsite.com. br/artigo/mostra_conteudo121416.asp?id_conteudo $=121416>$.

Pacheco, S., Botega, N. \& Silveira, G. (1996). Repercussões psicossociais em mulheres acometidas por câncer de mama. Rev. Méd., PUCRS, 6(3), 3-23.

Tubiana, M. \& Koscielny, S. (1999). The rationale for early diagnosis of câncer. Acta Oncol, 39, 295-303.

Urbanetz, A., Oliveira, L. \& Hatschbach, S. (1999). Análise crítica dos métodos diagnósticos do câncer inicial da mama. Jornal Brasileiro de Ginecologia, 102(1/2), 3-9.

Víctora, C. G., Knauth, D. R. \& Hassen. M. N. (2000). Pesquisa qualitativa em saúde: uma introdução ao tema. Porto Alegre: Tomo.

Recebido em outubro de 2004 Reformulado em junho de 2005 Aprovado em setembro de 2005

Psico-USF, v. 10, n. 2, p. 185-190, jul./dez. 2005 
Sobre os autores:

Marisa Campio Müller é doutora em Psicologia Clínica pela PUCSP, professora adjunta da PUCRS - Porto Alegre, orientadora desta Pesquisa, coordenadora do Grupo de Pesquisa intitulado Psicologia da Saúde do Programa de Pós-Graduação em Psicologia Clínica.

Antônio Luiz Frasson é médico, doutor em Medicina pela Universidade Federal do Rio de Janeiro, professor e coordenador do Centro de Mama do Hospital São Lucas da PUCRS, Mastologista.

Chana Kieling é graduanda do V semestre na PUCRS - Porto Alegre, bolsista de Iniciação Científica PIBIC/ CNPq desta pesquisa, membro do Grupo de Pesquisa Psicologia da Saúde do Programa de Pós-Graduação em Psicologia Clínica da PUCRS.

Fernanda Silva Hoffmann é mestranda em Psicologia Clínica da PUCRS - Porto Alegre, membro do Grupo de Pesquisa Psicologia da Saúde do Programa de Pós-Graduação em Psicologia Clínica da PUCRS.

Patrícia Fleck é mestranda em Psicologia Clínica da PUCRS - Porto Alegre, membro do Grupo de Pesquisa Psicologia da Saúde do Programa de Pós-Graduação em Psicologia Clínica da PUCRS, bolsista CAPES.

Hericka Zogbi é doutoranda em Psicologia Clínica da PUCRS - Porto Alegre, membro do Grupo de Pesquisa Psicologia da Saúde do Programa de Pós-Graduação em Psicologia Clínica da PUCRS, bolsista CNPq.

Adriana Zanona da Matta é mestranda em Psicologia Clínica da PUCRS - Porto Alegre, membro do Grupo de Pesquisa Psicologia da Saúde do Programa de Pós-Graduação em Psicologia Clínica da PUCRS, bolsista CAPES.

Joyce Werres é mestranda em Psicologia Clínica da PUCRS - Porto Alegre, membro do Grupo de Pesquisa Psicologia da Saúde do Programa de Pós-Graduação em Psicologia Clínica da PUCRS, bolsista CAPES. 